
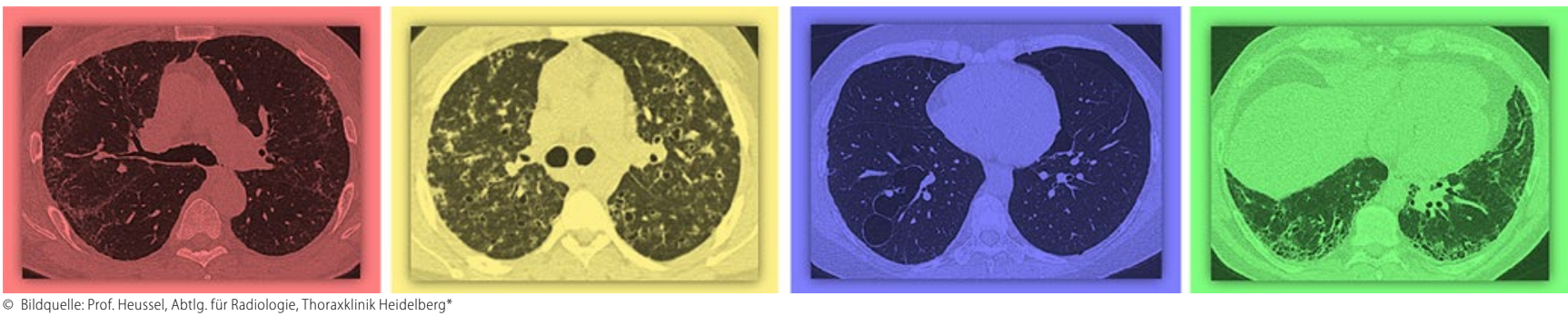

Interstitielle Lungenerkrankungen (ILD) in Deutschland

\title{
Das Register EXCITING-ILD stellt sich vor
}

\author{
Erkrankungsregister haben in den letzten Jahren neue Einsichten in die Versorgungsrealität \\ vieler chronischer Erkrankungen liefern können. Nun wurde ein Register zu interstitiellen \\ Lungenerkrankungen (ILD) in Deutschland etabliert, mit spannenden Aussichten.
}

In den letzten Jahren konnten in der Pneumologie mehrere sehr erfolgreiche Register etabliert werden [1, 2]. Im Vergleich zu klinischen Studien mit deutlich selektierten Populationen spiegeln Register die „Real-World-“Situation wider, da sie Patienten unabhängig von Begleiterkrankheiten bzw. -medikationen und in allen Schweregraden enthalten. Krankheitsregister sind klinischen Studien daher überlegen, weil sie die klinische Realität abbilden und helfen, die Studienergebnisse in die Praxis zu übertragen sowie nähere Einblicke in Krankheitsentitäten zu geben [1].

\section{Bisher wenig Daten zu ILDs in Deutschland}

Für die Gesamtheit der ILD liegen leider bisher - mit Ausnahme der IPF [2] - in Deutschland nur wenige alte Daten vor, die vor den derzeit gültigen Klassifikationen erhoben wurden [2]. Zudem wurden vornehmlich Daten zur Diagnostik gesammelt und erlauben keine Aussagen zur Prognose und prognostischen Faktoren, den Therapieformen und der Sozio- und Gesundheitsökonomie. Insbesondere im Hinblick auf die aktuelle Diskussion um ILDs, deren diversifizierter werdende Diagnostik und Therapie, aber auch auf gesundheitspolitische und -ökonomische Fragen kann uns ein aktuelles Register für ILDs helfen, diese Erkrankungen besser zu verstehen, Therapien zu optimieren und vor allem die Versorgung unserer Patienten zu verbessern.

Dies konnte bereits die erste Analyse des INSIGHTS-IPF-Registers sehr deutlich machen. Hier gelang es nicht nur, die Umsetzung der aktuellen IPF-Leitlinien nachzuvollziehen, sondern auch, die noch bestehenden Limitationen deutlich aufzuzeigen. Denn obwohl wir z.B. alle Lungensport, pulmonale Rehabilitation usw. propagieren, erhält nur eine sehr geringe Minderheit unserer IPF-Patienten eine solche Therapie [2].

Ziel des multizentrischen Registers EXCITING ist es also, tiefere Einblicke in die Charakteristika, das Management und vor allem die Prognose aller in Deutschland vorkommender ILDs unter klinischen Praxisbedingungen zu erlangen [4].

\section{Schon 250 Patienten erfasst}

In das Register wird das gesamte Spektrum der ILD bei Erwachsenen inkl. ILDs bekannter Ursachen (z.B. kollagenoseassoziiert), Granulomatosen, idiopathischer und anderer Formen (z.B. LAM) eingeschlossen. Erfasst werden die diagnostischen Prozeduren von ILDs, Prävalenzen, Inzidenzen, Prognose und prognostisch

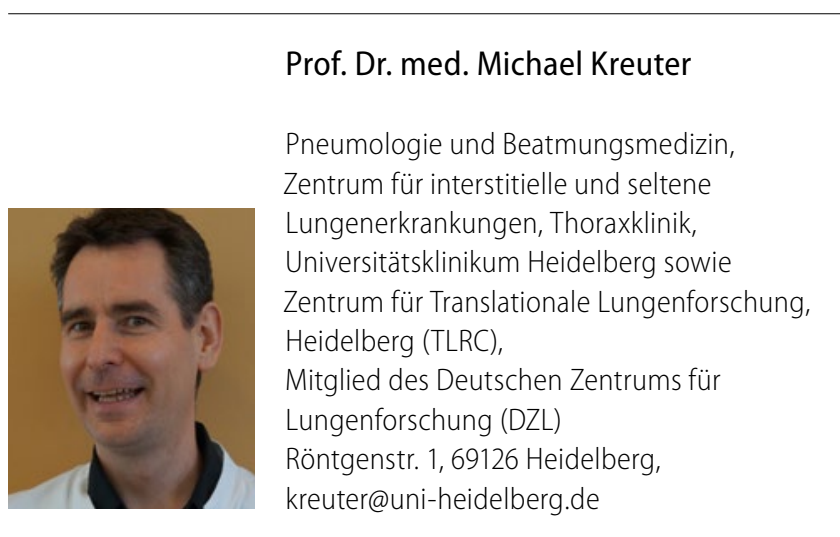

relevante Faktoren der ILDs, krankheitsbezogene Charakteristika, medikamentöse und nicht medikamentöse Therapien der verschiedenen Subtypen, wichtige Komorbiditäten sowie soziodemografische und gesundheitsökonomische Daten.

Das Register rekrutiert seit 2015 Patienten aus niedergelassenen pneumologischen Praxen, pneumologischen Fachabteilungen und spezialisierter ILD-Zentren und beobachtet sie bis zu 5 Jahre nach. Inzwischen wurden $>250$ Patienten eingeschlossen. Bisherige Interimsanalysen sind sehr vielversprechend, werden regelmäßig auf Kongressen vorgestellt und geben erstmals seit über 30 Jahren Einblicke in die Versorgungsrealität in Deutschland.

Hoffen wir, dass uns auch das EXCITING-ILD-Register ähnlich aufregende Einblicke geben wird wie andere Register.

\section{Literatur}

1. S. Gläser, D. Pittrow. Epidemiologie u. Register bei seltenen Lungenerkrankungen, in: Seltene Lungenerkrankungen. Springer Verlag Berlin, Heidelberg. 2016, 4-8.

2. Behr J, Kreuter M, Hoeper MH et al. Management of patients with idiopathic pulmonary fibrosis in clinical practice: INSIGHTS-IPF registry. Eur Respir J 2015; 46:186-96.

3. H. Schweisfurth, "Report by the Scientific Working Group for therapy of lung diseases: German fibrosis register with initial results," Pneumologie, 50, no. 12, pp. 899-901, 1996 (German).

4. Kreuter M, Herth FJF, Wacker M et al. Exploring clinical and epidemiological characteristics of interstitial lung diseases (EXCITING-ILD) - rationale, aims and design of a nationwide prospective registry. Biomed Res Int, 2015:123876 